\title{
CHOICES FROM FINITE SETS AND CHOICES OF FINITE SUBSETS
}

\author{
MARTIN M. ZUCKERMAN
}

\begin{abstract}
In set theory without the axiom of choice we prove a consistency result involving certain "finite versions" of the axiom of choice. Assume that it is possible to select a nonempty finite subset from each nonempty set. We determine sets $Z$, of integers, which have the property that $n \in Z$ is a necessary and sufficient condition for the possibility of choosing an element from every $n$-element set. Given any nonempty set $P$ of primes, the set $Z_{p}$, consisting of integers which are not "linear combinations" of primes of $P$, is such a set $Z$.
\end{abstract}

1. Introduction. Let $\sigma$ be the system of set theory of [4]. This is a system of the Gödel-Bernays type which permits the existence of urelemente (objects in the domain, but not the range of the $\epsilon$-relation) and which does not include among its axioms the axiom of choice.

By AC (the axiom of choice) we mean the statement of $\sigma$ : "For every nonempty set $X$ of nonempty sets there is a function $f$ defined on $X$ such that $f(x) \in x$ for each $x \in X$." We consider various "finite versions" of the axiom of choice. Let FS be the statement: "For every nonempty set $X$ of nonempty sets there is a function $f$ defined on $X$ such that $f(x)$ is a nonempty finite ${ }^{1}$ subset of $x$ for each $x \in X$." Identify nonnegative integers with finite Von Neumann ordinals. For each positive integer $n$ let $C(n)$ be the statement: "For every nonempty set $X$ of $n$-element sets there is a function $f$ defined on $X$ such that $f(x) \in x$ for each $x \in X$." Thus $C(1)$ is a (trivial) theorem of $\sigma$. For a subset $Z=\left\{z_{0}, z_{1}, \cdots, z_{k-1}\right\}$ of integers $\geqq 2$, let $C(Z)$ be the conjunction of the statements $C\left(z_{i}\right), i \in k$.

We are concerned with the following problem. Assume $\sigma$ is consistent. For which sets $Z$ of integers $\geqq 2$ is the set of axioms

$$
\sigma \cup\{\neg \mathrm{AC}, \mathrm{FS},(\forall n \geqq 2)(C(n) \leftrightarrow n \in Z)\}
$$

also consistent. Let $Z$ be the set of sets $Z$ for which (1) holds.

Received by the editors March 14, 1969.

AMS 1970 subject classifications. Primary 02K05, 02K20, 02A20, 02A25; Secondary $05 \mathrm{~A} 05$.

Key words and phrases. Axiom of choice for $n$-element sets, multiple choice axioms, Fraenkel-Mostowski models, relative consistency.

1 A set $A$ is finite iff every nonempty set of subsets of $A$ has a maximal element with respect to inclusion; otherwise, $A$ is infinite.

Copyright (c) 1971, American Mathematical Society 
For each positive integer $n$, let $I_{n}$ be the set of integers $\geqq n$. Theorem 6 of [2] shows that $I_{2} \in Z$; Theorem 1 of [6] implies that 0 (the empty set) is in $\mathrm{Z}$. If $P$ is any nonempty set of primes, let Lin Comb $P$ be the set of positive integers of the form $k_{0} p_{0}+k_{1} p_{1}$ $+\cdots+k_{s-1} p_{s-1}$, where $s \in I_{1}$ and for each $i \in s, k_{i} \in I_{0}$ and $p_{i} \in P$. The main result of the present paper shows that if $P$ is any (nonempty) set of primes and if $Y=I_{2} \backslash \operatorname{Lin} \operatorname{Comb} P$, then $Y \in Z^{2}{ }^{2}$ It is well known that if $P$ contains more than two primes and if $p$ and $q$ are the two smallest primes of $P$, then $I_{(p-1)(q-1)} \subseteq$ Lin Comb $P$. Thus we need only consider finite sets of primes.

We prove our theorem by constructing a Fraenkel-Mostowski model of set theory; we employ a variation of the technique used in [2] and [6]. As an immediate corollary of our theorem we have a direct proof of the necessity of Mostowski's condition (M) (defined below) for an implication of the form $C(Z) \rightarrow C(n)$ to be provable in $\sigma$.

\section{The model.}

Theorem. Assume $\sigma$ is consistent. Let $P$ be any nonempty finite set of primes and let $Y=I_{2} \backslash$ Lin Comb $P$. Then there is a model of

$$
\sigma \cup\{\neg \mathrm{AC}, \mathrm{FS},(\forall n \geqq 2)(C(n) \leftrightarrow n \in Y)\} .^{3}
$$

Proof. Let $\sigma^{*}$ be $\sigma$ together with the axiom of choice and an axiom asserting the existence of a denumerable set of urelemente. $\sigma^{*}$ is relatively consistent with $\sigma$ (see [2, pp. 478-479]); we shall work within $\sigma^{*}$.

Let $\mathfrak{T}$ be a denumerable set of urelemente. Define $\mathfrak{T}_{0}$ to be $\mathfrak{T}$ and for each ordinal number $\eta>0$, let $\mathfrak{T}_{\eta}=\mathfrak{N} \cup \cup\left\{\rho\left(\mathfrak{T}_{\mu}\right): \mu \in \eta\right\}$.

Let $\mathrm{G}_{0}$ be the group of all one-one transformations of $\mathfrak{N}$ onto itself. By transfinite induction, if $x \in \mathfrak{M}_{\eta} \backslash \cup \mathfrak{M}_{\zeta}(\zeta \in \eta)$ for some $\eta>0$ and if $\phi \in \mathcal{G}_{0}$, we "extend" $\phi$ by letting $\phi(x)=\{\phi(y): y \in x\}$.

Let $P=\left\{p_{0}, p_{1}, \cdots, p_{s-1}\right\}$. Let $T$ be the subset of $I_{0} \times I_{0}$ consisting of all ordered pairs $\langle i, t\rangle$, where $i$ ranges over $I_{0}$ and where $t \in p_{j}$ if $i \equiv j(\bmod s)$. Since $T$ is denumerable, there is a one-one correspondence between $T$ and $\mathfrak{T}$. With respect to any such one-one correspondence, let $\mathfrak{m}_{i, t}$ be the member of $\mathfrak{T}($ which corresponds to $\langle i, t\rangle$.

2 This result was also obtained, independently, by D. Pincus.

${ }^{3}$ It was remarked in [2, p. 478] that using Mendelson's technique of [3], Theorem 6 of [2] can be proved with "Gödel's system $A, B, C$ " replacing " $\sigma$ " in the hypothesis or conclusion. The same remark applies to Theorem 1 of [6] and to our present theorem. 
For each $i \in I_{0}$ and $j \in s$, let $q_{i}=p_{j}$ if $i \equiv j(\bmod s)$ and let $\operatorname{Tr}^{(i)}$ $=\left\{\mathfrak{m}_{i, t}: t \in q_{i}\right\}$. Then $\mathfrak{N}$ is the pairwise disjoint union of the $\mathfrak{N}^{(i)}$, $i \in I_{0}$. Let $\chi_{i}$ be the element of $g_{0}$ which maps $m_{j, t}$ into itself for $j \neq i$, and which maps $\mathfrak{m}_{i, t}$ into $\mathfrak{m}_{i, u}$ for $t, u \in q_{i}$ and $u \equiv t+1\left(\bmod q_{i}\right)$. Let $\mathcal{G}_{1}$ be the subgroup of $\mathcal{G}_{0}$ generated by $\left\{\chi_{i}: i \in I_{0}\right\}$. If $Z$ is a finite set of integers and if $\phi \in \mathcal{G}_{1}$; then $\phi$ is said to be $Z$-identical if $\phi\left(\mathfrak{m}_{i, t}\right)=\mathfrak{m}_{i, t}$ for every pair $\langle i, t\rangle$ for which $i \in Z$. If $x \in \mathbb{M}_{\eta}$ for some ordinal number $\eta$, then $x$ is said to be $Z$-symmetric if $\phi(x)=x$ for every $Z$-identical $\phi \in \mathcal{G}_{1}$.

For each ordinal number $\eta$, we define $\kappa_{\eta}$ by transfinite induction: $\mathfrak{K}_{0}=\mathfrak{M} \cup\{0\}$, and for each $\eta>0$,

$$
\begin{aligned}
x \in \mathcal{K}_{\eta} & \leftrightarrow(\forall y \in x)(\exists \xi \in \eta)\left(y \in \mathcal{K}_{\xi}\right) \\
& \wedge\left(x \text { is } Z \text {-symmetric with respect to some finite set } Z \subset I_{2}\right) .
\end{aligned}
$$

$x$ is said to be an $\mathfrak{N}$-element if there exists an ordinal number $\eta$ such that $x \in \mathscr{K}_{\eta}$. A class $X$ is called an $\Re$-class if every element of $X$ is an $\pi$-element and if there is a finite set $Z$ of integers with the property that for every $Z$-identical $\phi \in \mathcal{G}_{1}, \phi(y) \in X$ for every $y \in X$. If $X$ and $Y$ are classes, define $X \in_{\Re} Y$ to be true iff $X$ is an গ⿰亻-element, $Y$ is an $\Re$-class and $X \in Y$. Then, if we interpret $\sigma$ in $\sigma^{*}$ by replacing the primitive notions "element," "class," " $\in$," and " 0 " by the no-

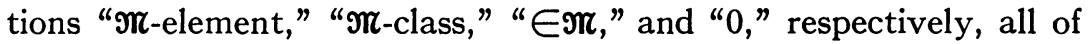
the axioms and theorems of $\sigma$ will become theorems of $\sigma^{*}$.

For a discussion of Fraenkel-Mostowski models, and in particular for a verification of some of the axioms in these models, see [4] and [5]. [2] and [6] discuss absoluteness in these models. The verification of FS (called " $Z(\infty)$ ") is carried out in [2] and applies to the present model.

We now show that if $n \in \operatorname{Lin} \operatorname{Comb} P$, then $C(n)$ is false in the model. For each such $n$, let $k_{0}, k_{1}, \cdots, k_{s-1}$ be any integers for which $n=k_{0} p_{0}+k_{1} p_{1}+\cdots+k_{s-1} p_{s-1}$. Delete the zero terms and write this sum as

$$
n=k_{i_{0}} p_{i_{0}}+k_{i_{1}} p_{i_{1}}+\cdots+k_{i_{v}} p_{i_{v}}, \quad 0 \leqq i_{0}<i_{1}<\cdots<i_{v}<s .
$$

Let

$$
x_{n}=\left\{\bigcup_{j=0}^{0} \bigcup_{l=m k_{i_{j}}}^{(m+1) k_{i_{i}}-1} \mathfrak{N}^{\left(i_{j}+l_{0}\right)}: m \in I_{0}\right\} .
$$

Then $x_{n}$ is a set of $n$-element sets. Clearly, $x_{n} \in \mathfrak{M}_{2}$; moreover, $x_{n}$ is 0 -symmetric, and it is, consequently, in $\Re_{2}$. Suppose that $f$ is a choice 
function on $x_{n}$. Then $f$ must be $Z$-symmetric for some finite $Z \subset I_{0}$. For $m \in I_{0}$, let

$$
\begin{aligned}
R_{m}=\left\{i_{j}+l s: l=m k_{i}, m k_{i}+1, \cdots,(m+1) k_{i_{j}}-1\right. & ; \\
j & =0,1, \cdots, v\} .
\end{aligned}
$$

Let $m_{0}$ be the smallest integer $m$ for which $Z \cap R_{m}=0$. Then for each $r \in R_{m_{0}}, \chi_{r}$ is $Z$-identical and, hence, $\chi_{r}(f)=f$. Let

$$
\left(x_{n}\right)_{m_{0}}=\bigcup_{j=0}^{0} \bigcup_{l=m_{0} k_{i_{j}}}^{\left(m_{0}+1\right) k_{i_{j}}-1} \mathfrak{T}^{\left(i_{j}+l s\right)} .
$$

Now since $f\left(\left(x_{n}\right)_{m_{0}}\right)=\left(x_{n}\right)_{m_{0}}$ and since the $\mathfrak{T}^{(j)}$ are pairwise disjoint, it follows that $f\left(\left(x_{n}\right)_{m_{0}}\right) \in \Re^{(r)}$ for some unique $r \in R_{m_{0}}$, and, consequently, that $f\left(\left(x_{n}\right)_{m_{0}}\right)=\mathfrak{m}_{r, t}$ for some $t \in q_{r}$. Thus $\left\langle\left(x_{n}\right)_{m_{0}}, \mathfrak{m}_{r, t}\right\rangle$ $\in f$. But then $\left\langle\left(x_{n}\right)_{m_{0}}, \chi_{r}\left(\mathfrak{m}_{r, t}\right)\right\rangle=\chi_{r}\left(\left\langle\left(x_{n}\right)_{m_{0}}, \mathfrak{m}_{r, t}\right\rangle\right) \in \chi_{r}(f)=f$; since $\chi_{r}\left(\mathfrak{m}_{r, t}\right) \neq \mathfrak{m}_{r, t}, f$ cannot be a function.

It remains to show that if $n \notin \operatorname{Lin} \operatorname{Comb} P$, then $C(n)$ is true in the model. Let $X$ be a nonempty $\Re$-set of $n$-element sets $x$. Then $X$ $\in \mathcal{K}_{\alpha+1} \backslash \mathcal{K}_{\alpha}$ for some $\alpha \geqq 1$, and $X$ is $Z$-symmetric for some finite $Z \subset I_{0}$. Let $\mathrm{g}^{Z}$ be the subgroup of $\mathcal{G}_{1}$ consisting of all $Z$-identical maps. Then for each $\phi \in \mathcal{G}^{Z}, X=\phi(X)=\{\phi(x): x \in X\}$.

We first show that

$$
\begin{aligned}
& \text { for each } x \in X \text {, there is an } a \in x \text { with the } \\
& \text { property that whenever } \phi, \psi \in \mathcal{G}^{Z} \text { and } \\
& \phi(x)=\psi(x), \text { then } \phi(a)=\psi(a) .
\end{aligned}
$$

Equivalently, we show that for each $x \in X$ there is an $a \in x$ with respect to which $\phi \in \mathcal{G}^{Z}$ and $\phi(x)=x$ together imply $\phi(a)=a$.

Suppose $X \in \mathfrak{K}_{2} \backslash \mathfrak{K}_{1}$ and $x \in X$. Then the elements of $x$ are in $\mathfrak{K}_{0}$. If $0 \in x$, then $\phi(0)=0$ for every $\phi \in \mathcal{G}^{Z}$; if $\mathfrak{m}_{i, t} \in x$ for some $i \in Z$, then $\phi\left(\mathfrak{m}_{i, t}\right)=\mathfrak{m}_{i, t}$ for every $\phi \in \mathcal{G}^{Z}$. Otherwise, $x$ consists of $n$ urelemente in $\mathfrak{M}_{0} \backslash \bigcup_{i \in Z} \mathfrak{T}^{(i)}$. Now each $\mathfrak{T}^{(j)}$ consists of $q_{j}$ elements for some $q_{j} \in P$, whereas $n \notin \operatorname{Lin}$ Comb $P$. Thus for some $j, x \cap \Re^{(j)}$ is a nonempty proper subset of $\mathfrak{T}^{(j)}$. Pick any such $j$ and any $\mathfrak{m}_{j, t} \in x$. For any $\phi \in \mathcal{G}^{Z} \backslash \mathcal{G}^{|j|}$, if $\phi\left(\mathfrak{m}_{j, t}\right) \neq \mathfrak{m}_{j, t}$, then $\phi\left(\mathfrak{m}_{j, t}\right)=\mathfrak{m}_{j, u}$ for some $u \in s \backslash\{t\}$. Thus $\phi\left(x \cap \Re^{(j)}\right)=\left\{\mathfrak{m}_{j, v}\right.$ : some $\mathfrak{m}_{j, w} \in x$ and $v-w \equiv u-t$ $\left.\left(\bmod q_{j}\right)\right\} \neq x \cap \Re^{(j)}$, and since $\phi\left(x \backslash \mathfrak{N}^{(j)}\right) \cap \Re^{(j)}=0$, it follows that $\phi(x) \neq x$.

For any $\pi$-set $Y$, let $\Sigma_{0}(Y)=Y$ and for ordinal numbers $\xi>0$ let $\Sigma_{\xi}(Y)=Y \cup\left\{y\right.$ : for some $\eta<\xi$ and some $\left.z \in \Sigma_{\eta}(Y), y \in z\right\}$. (Properties of the $\Sigma_{\xi}(Y)$ are discussed in [4].) 
Let $\Sigma(Y)=\left\{z: z \in \Sigma_{\xi}(Y)\right.$ for some ordinal number $\left.\xi\right\}$.

An element of $\varkappa_{0} \cap \Sigma(Y)$ will be called a progenitor of $Y$.

A transfinite induction argument shows that for any $\phi \in \mathcal{g}^{Z}$ and IT-set $Y$, a necessary condition that $\phi(Y)=Y^{\prime}$ is that $Y$ and $Y^{\prime}$ have the same number of progenitors in each $\Re^{(j)}, j \in I_{0}$.

Now let $X \in \Re_{\alpha+1} \backslash \Re_{\alpha}$ for $\alpha>1$, and let $x \in X$. We may assume that either $x \cap \varkappa_{0}=0$ or else that the number of elements of $x \cap \varkappa_{0}$ is in Lin Comb $P$; otherwise, the previous argument applies. Suppose that for $\phi \in \mathcal{G}^{Z}$ we have $\phi(x)=x$ but $\phi(a) \neq a$ for every $a \in x$. Let $a_{1} \in x \backslash \mathcal{K}_{0}$. Then, surely, for some progenitor $\mathfrak{m}_{i, t}$ of $a_{1}$ (and hence of $\left.x\right), \phi\left(\mathfrak{m}_{i, t}\right)$ $\neq \mathfrak{m}_{i, t}$. Now for every $a \in x, \phi(a) \in x$. Thus for some positive integer $h_{i_{1}}, x \backslash \mathcal{K}_{0}$ must contain $h_{i_{1}} q_{i_{1}}$ elements, with progenitors in $\Re^{\left(i_{1}\right)}$, which are cyclically permuted by $\phi$. Since $n \notin \operatorname{Lin}$ Comb $P$, and $x$ contains $n$ elements, there must be an element $a_{2}$ in $x \backslash \mathcal{K}_{0}$ distinct from these $h_{i_{1}} q_{i_{1}}$ elements. Repeat the above argument for $a_{2}$ and obtain $h_{i_{2}} q_{i_{2}}$ elements of $x \backslash \mathfrak{K}_{0}$ for $q_{i_{2}} \in P, h_{i_{2}} \geqq 1$. These new elements are distinct from the previous ones; again, these $h_{i_{1}} q_{i_{1}}+h_{i_{2}} q_{i_{2}}$ elements cannot exhaust $x \backslash \mathcal{K}_{0}$. Clearly, in a finite number, $r$, of steps we will obtain $\sum_{j=1}^{r} h_{i j} q_{i j}$ distinct elements of $x \backslash \mathcal{K}_{0}$, where for $j=1$, $2, \cdots, r, h_{i_{j}} \geqq 1$ and the $q_{i_{j}}$ are (not necessarily distinct) primes of $P$, and where $\sum_{j=1}^{r} h_{i j} q_{i j}>n$. This contradicts the assumption that $x$ has $n$ elements and completes the proof of (2).

Define the relation $R$ on $X$ by $x_{1} R x_{2}$ iff there is some $\phi \in g^{z}$ for which $\phi\left(x_{1}\right)=x_{2}$. $R$ is obviously an equivalence relation on $X$. Choose an element $x_{C}$ from each cell $C$ of the partition $X / R$; choose an element $a_{C}$ in each such chosen $x_{C}$ with the property that whenever $\phi\left(x_{C}\right)=\psi\left(x_{C}\right), \phi, \quad \psi \in \mathcal{S}^{Z}$, then $\phi\left(a_{C}\right)=\psi\left(a_{C}\right)$. Let $f_{C}$ $=\left\{\left\langle\phi\left(x_{C}\right), \phi\left(a_{C}\right)\right\rangle: \phi \in \mathcal{G}^{Z}\right\}$. Each such $f_{C}$ is a function with domain $C$. Clearly $f_{C} \in \mathfrak{N}_{\alpha+3}$. Moreover, $f_{C}$ is $Z$-symmetric because for $\psi \in \mathcal{G}^{Z}, \psi\left(f_{C}\right)=\left\{\left\langle\psi \phi\left(x_{C}\right), \psi \phi\left(a_{C}\right)\right\rangle: \phi \in \mathcal{G}^{Z}\right\}=f_{c}$. Thus $f_{C} \in \mathcal{K}_{\alpha+3}$. Let $f=U_{C \in X / R} f_{C} ; f$ is also $Z$-symmetric and is in $\mathcal{K}_{\alpha+3} . f$ is the desired choice function for $X$.

A finite subset $Z \subset I_{2}$ and an integer $n \in I_{2}$ are said to satisfy condition (M) iff for every finite set $P$ of primes, $n \in$ Lin Comb $P$ implies $Z \cap L$ in Comb $P \neq 0$.

In [5], Mostowski proves the necessity of condition $(M)$ for the implication $C(Z) \rightarrow C(n)$ to hold in $\sigma$ by first considering the following condition $(\mathrm{K})$, which we proceed to define.

For $n \geqq 1$, let $S_{n}$ be the symmetric group on $\{1,2, \cdots, n\}$. If $g$ is a subgroup of $\delta_{n}$, if $1 \leqq k \leqq n$, and if $\phi(k)=k$ for every $\phi \in \mathcal{G}$, we say that $k$ is a fixed-point of $\mathcal{G}$. For any group $\mathcal{G}$, let $\mathcal{G}^{\omega}$ denote the group whose elements are those infinite sequences, $\left\langle g_{1}, g_{2}, \ldots\right\rangle$, whose terms 
belong to $\mathcal{G}$ and which are such that almost all of the $g_{n}$ are equal to the unity of $\mathcal{G}$; multiplication in $\mathcal{G}^{\omega}$ is defined by termwise multiplication in $\mathrm{G}$. A finite subset $Z$ of $I_{1}$ and an $n \in I_{1}$ satisfy condition (K) if for every subgroup $\mathcal{G}$ of $\delta_{n}$ without fixed-points there is a group ${ }^{*} C \mathcal{S}^{\omega}$ and a finite number $r$ of (not necessarily different) proper subgroups $\mathfrak{L}_{1}, \mathfrak{L}_{2}, \cdots, \mathfrak{L}_{r}$ of $\mathfrak{H C}$ such that

$$
\sum_{i=1}^{r} \operatorname{Ind}\left(\mathcal{H} / \mathscr{L}_{i}\right) \in Z .
$$

The model constructed in [5] proves that $(\mathrm{K})$ is necessary for $C(Z)$ $\rightarrow C(n)$ to hold in $\sigma$; it is then shown that if $Z$ and $n$ satisfy $(\mathrm{K})$ they also satisfy $(M)$.

As an immediate consequence of our theorem we obtain:

COROLlaRY 1. Condition (M) is necessary for the implication $(\mathrm{FS} \wedge C(Z)) \rightarrow C(n)$ to hold in $\sigma$.

By taking $P$ to consist of a single prime we obtain:

COROLlaRY 2. For each prime $p$, there is a model for $\sigma \cup\{7 \mathrm{AC}, \mathrm{FS},(\forall n \geqq 2)(C(n) \leftrightarrow(n$ is not a multiple of $p)\}$.

Thus there is a model $\Re($ in which the set $Z$ of integers $n$ for which $C(n)$ is true in $\mathfrak{T}$ as well as the set $Y$ of $n$ for which $C(n)$ is false in $\mathfrak{N}$, are both infinite.

\section{REFERENCES}

1. K. Gödel, The consistency of the axiom of choice and of the generalized continuum hypothesis with the axioms of set theory, 6th ed., Ann. of Math. Studies, no. 3; Princeton Univ. Press, Princeton, N. J., 1964.

2. A. Lévy, Axioms of multiple choice, Fund. Math. 50 (1962), 475-483.

3. E. Mendelson, The independence of a weak axiom of choice, J. Symbolic Logic 21 (1956), 350-366. MR 18, 864.

4. A. Mostowski, Über die Unabhängigkeit des Wohlordnungssätzes vom Ordnungsprinzip, Fund. Math. 32 (1939), 201-252. $8,3$.

5. —, Axiom of choice for finite sets, Fund. Math. 33 (1945), 137-168. MR

6. M. M. Zuckerman, Multiple choice axioms, Proc. Sympos. Pure Math., vol. 13. Amer. Math. Soc., Providence, R. I., 1970. 10031

City College of the City University of New York, New York, New York 